\title{
Editorial
}

\section{Low-Dimensional Semiconductor Structures for Optoelectronic Applications}

\author{
Wei Lu, ${ }^{1}$ Hong Chen, ${ }^{2}$ Weida Hu, ${ }^{1}$ Guofeng Song, ${ }^{3}$ Wen Lei, ${ }^{4}$ and Ying Fu ${ }^{5}$ \\ ${ }^{1}$ Shanghai Institute of Technical Physics, Chinese Academy of Sciences, Shanghai 200083, China \\ ${ }^{2}$ The Institute of Physics, Chinese Academy of Sciences, Beijing 100190, China \\ ${ }^{3}$ The Institute of Semiconductors, Chinese Academy of Sciences, Beijing 200083, China \\ ${ }^{4}$ University of Western Australia, Crawley, WA 6009, Australia \\ ${ }^{5}$ The Royal Institute of Technology (KTH), 13640 Stockholm, Sweden
}

Correspondence should be addressed to Wei Lu; luwei@mail.sitp.ac.cn

Received 15 February 2015; Accepted 15 February 2015

Copyright (C) 2015 Wei Lu et al. This is an open access article distributed under the Creative Commons Attribution License, which permits unrestricted use, distribution, and reproduction in any medium, provided the original work is properly cited.

A truly extraordinary research effort is pressed to develop a full understanding of the properties of matters at the nanoscale and its possible applications. The aim of the special issue is to include the recent advances in new and original theoretical, experimental, and/or simulation works in narrow-gap, nanoscaled, and low-dimensional structures for infrared detectors, solar cells, and transistors.

Among a large number of submissions, we have selected 10 papers for publication in the special issue. The work reported by $\mathrm{H}$. Cui et al. is believed to be very useful in measuring the minority carrier lifetime of $\mathrm{HgCdTe}$ photodetector by using optical and electrical methods. The paper by Y. Liu et al. reports an experimental work for determining temperature-dependent stress state in thin $\mathrm{AlGaN}$ layer of AlGaN/GaN HEMT heterostructures by near-resonant Raman scattering. The theoretical modeling is in good agreement with the measurements. M. J. Wang et al. and W. W. Wang et al. published two papers on InGaAs/GaAs quantum dot/quantum well infrared photodetector. Both papers are highly important for the design, fabrication, and characterization of quantum dot/quantum well infrared photodetectors. A two-dimensional simulation for effects of structure parameters on shot channel effects of lowdimensional nanoscaled inversion-mode InGaAs MOSFETs has been investigated in detail by $\mathrm{C}$. $\mathrm{H}$. Yu et al. The paper by
L. Li and D. Xiong reports a mathematical model for investigating photoresponse of long-wavelength $\mathrm{AlGaAs} / \mathrm{GaAs}$ quantum cascade detectors. The theoretical results for simulating photoresponse of $\mathrm{AlGaAs} / \mathrm{GaAs}$ detectors could be of immense importance for the designing of AlGaAs/GaAsbased optoelectronic devices. An interesting work on modeling and design of graphene GaAs junction solar cell reported by Y. Kuang et al. has also been considered for publication. D. Yin et al. report on synthesis, characterization, and photoluminescence on the glass doped with AgInS2 nanocrystals. The paper by F. Liu et al. presents a detailed investigation on shell thickness-dependent strain distributions of confined $\mathrm{Au} / \mathrm{Ag}$ and $\mathrm{Ag} / \mathrm{Au}$ core-shell nanoparticles, providing an effective method to manipulate the strain distributions of the $\mathrm{Au} / \mathrm{Ag}$ and $\mathrm{Ag} / \mathrm{Au}$ nanoparticles by tuning the thickness of the shell. The paper by G. Shanmuganathan and I. B. S. Banu reports influence of codoping on the optical properties of $\mathrm{ZnO}$ thin films synthesized on glass substrate by chemical bath deposition method, confirming that codoped $\mathrm{ZnO}$ thin films can be suitable candidates for antireflecting coating and optoelectronic devices. It is believed that the special issue will be of significant interest to the scientists and researchers working in the areas related to the narrow-gap semiconductors and low-dimensional structures for optoelectronic applications. 


\section{Acknowledgments}

We would like to thank all the authors and coauthors who submitted their papers to the special issue.

Wei Lu

Hong Chen

Weida $\mathrm{Hu}$

Guofeng Song

Wen Lei

Ying Fu 

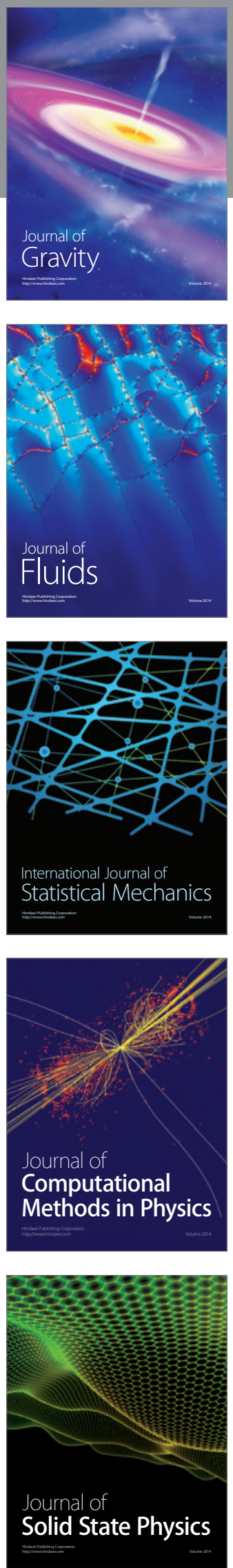

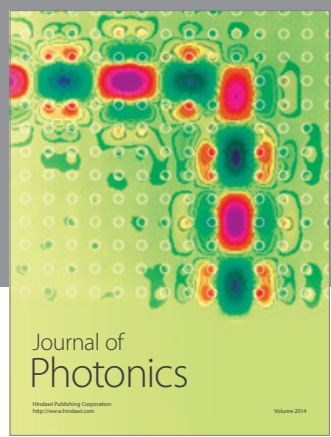

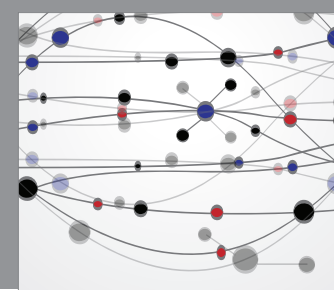

The Scientific World Journal

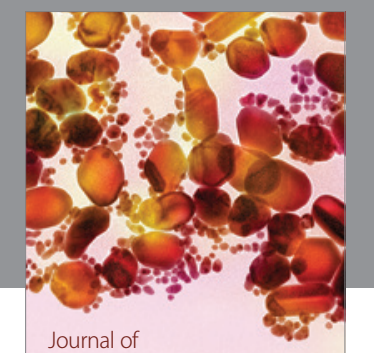

Soft Matter
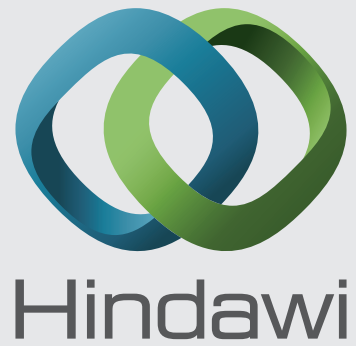

Submit your manuscripts at

http://www.hindawi.com
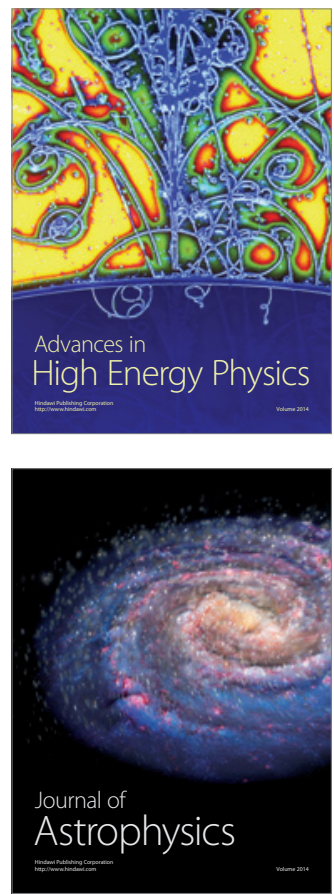
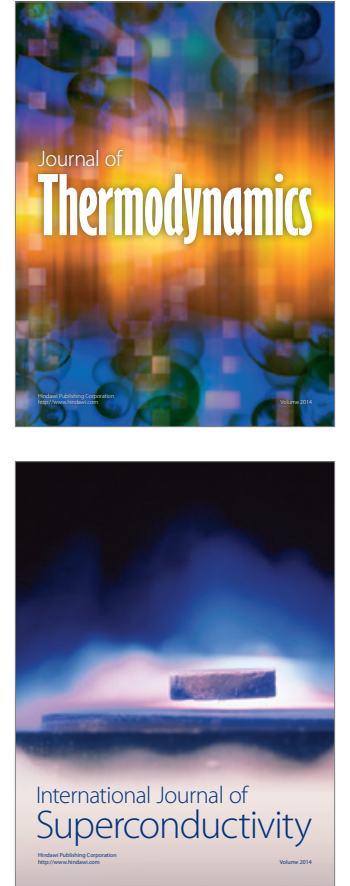
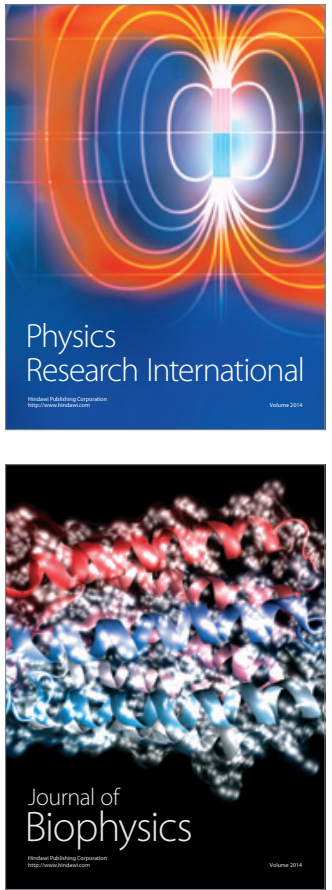
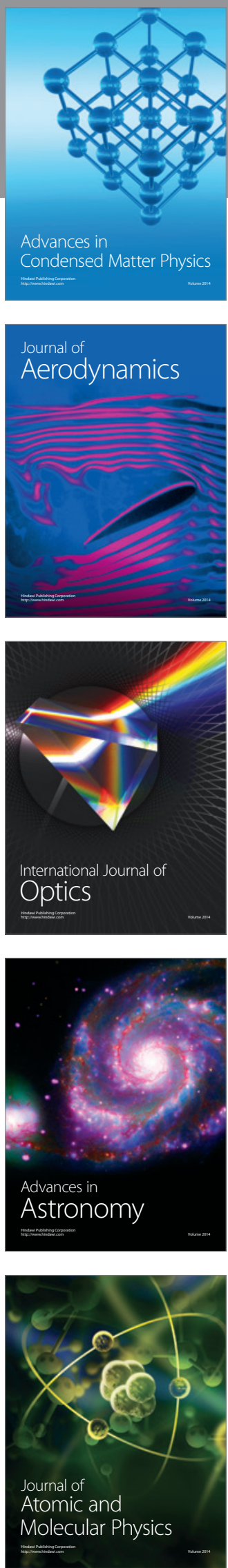